\title{
Procesos y tendencias de la urbanización en el noreste mexicano
}

\section{Processes and tendencies of urbanization in northeastern Mexico}

\author{
García Ortega, Roberto, Socorro Arzaluz Solano y Jesús \\ Manuel Fitch Osuna (coords.) (2009), TERRITORIO Y CIUDADES EN EL \\ noreste de MÉXICO al inicio del Siglo XXI, El Colegio de la \\ Frontera Norte-Miguel Ángel Porrúa, México, 354 PP., \\ ISBN: 978-607-40 I-I 94-4.
}

El libro que reseñamos surgió de un seminario internacional organizado por El Colegio de la Frontera Norte, Dirección General Noreste en Monterrey, y la Facultad de Arquitectura de la Universidad Autónoma de Nuevo León (UANL) en 2006.

A partir de distintas disciplinas que se vinculan directa e indirectamente con el campo de los estudios urbanos, un grupo de especialistas (algunos reconocidos en su trayectoria y otros investigadores en formación de gran calidad) abordan el fenómeno de la urbanización en la región noreste de México. Se trata de los trabajos de 12 autores: nueve son profesores e investigadores en la UANL (buena parte en las divisiones de pre y posgrado de la Facultad de Arquitectura de esta casa de estudios); tres desempeńan su labor como investigadores en El Colegio de la Frontera Norte (dos de ellos coordinadores de este libro); asimismo, la obra incorpora a dos profesores-investigadores, uno de la Texas A\&M University y otro de El Colegio de México.

En razón de lo anterior, la obra conjuga campos de conocimiento provenientes de la historia, la filosofía, la sociología, la psicología social, la economía, la geografía, la arquitectura, el urbanismo y el ordenamiento territorial. Las reflexiones van de lo general a lo particular, y comienzan con una revisión de la dinámica sociodemográfica de la urbanización en todo el país en los últimos 25 años, para arribar más tarde a la explicación de procesos específicos en el Área Metropolitana de Monterrey (AMM).

De esta manera, el propósito general del libro es mostrar el abanico de problemáticas que pueden abordarse para analizar el fenómeno urbano-metropolitano, de manera que se presentan diversas cuestiones, desde las físico-espaciales, sociales, políticas, históricas, demográficas, económicas y ambientales hasta las teóricas, que se imprimen en un territorio específico. Por lo anterior, y en función de presentar los argumentos de 
manera sistematizada, la estructura de contenido se organiza en 11 textos distribuidos en tres partes, de acuerdo con tres escalas o ámbitos territoriales, respectivamente: el ámbito nacional, la región noreste y el AMM.

La primera parte se compone sólo de un ensayo cuyo autor es Gustavo Garza Villarreal. El texto abre el contenido del libro, con el propósito de introducir al lector en la discusión a nivel macro respecto del fenómeno de la urbanización en México. El autor parte de la hipótesis que establece un vínculo histórico-estructural entre el desarrollo económico y la urbanización; por ello, metodológicamente propone como cronología la evolución económica nacional en tres etapas, de acuerdo con los años de la información censal, a saber: década perdida 1980-1990, recuperación relativa, 1990-1995 y recesión cíclica 1995-2005. A su vez distingue dos unidades de análisis:

1. Ocho regiones geoeconómicas, observando la población total y urbana por regiones mediante los incrementos poblacionales absolutos y relativos, para detectar cambios en la dinámica diferencial de las regiones en México.

2. El sistema de ciudades agrupadas según su tamaño (pequeño, mediano, grande), examinando los niveles de concentración demográfica por ciudades, y con ello las tendencias de la distribución de la población dentro de la jerarquía urbana nacional.

De acuerdo con la periodización sugerida, el autor hace comparaciones entre las regiones y sistemas urbanos, de las que conviene destacar las siguientes: un primer sondeo indica que en el cuarto de siglo transcurrido entre 1980 y 2005 se observa el inicio de modificaciones significativas en la distribución de la población total y urbana nacional en contra de las regiones centro-este y centro-oeste, y a favor del norte y sureste, sobre todo en el último periodo. En segundo lugar, advierte que el elevado crecimiento absoluto y relativo de la población urbana nacional no implicará necesariamente el tránsito hacia una jerarquía urbana más equilibrada, sino que podría significar el cambio de la concentración de una a unas cuantas metrópolis, así como el surgimiento de nuevos ámbitos de concentración de tipo megalopolitano o de regiones urbanas policéntricas (pp. 35, 38), situación que no es privativa de los países en proceso de desarrollo, sino que suele existir en todas las economías de corte capitalista (p. 42).

Con estas y otras conclusiones, el vínculo entre el desarrollo económico y la urbanización queda demostrado, por lo que según Garza es seguro esperar que el proceso de concentración de población en tres regiones urbanas policéntricas sea el resultado de la aglomeración territorial 
del proceso productivo (p. 53). En este sentido, el autor afirma que cualquier intento de planeación territorial debe, sin duda, partir del análisis de la dinámica macroeconómica nacional.

$\mathrm{Al}$ respecto, conviene comentar que si bien el texto atiende el ámbito nacional, se señalan decisiones de política económica de los últimos 10 años (por ejemplo, la puesta en marcha del Tratado de Libre Comercio de América del Norte, TLCAN), que desencadenaron fenómenos como el impulso de la industria maquiladora, que ha tenido efecto en la dinámica demográfica, en particular de las regiones del norte.

La segunda parte se compone de cinco textos que abordan tanto la dinámica actual como las circunstancias históricas de la región fronteriza noreste y sus principales ciudades, y presenta, sobre todo en los dos primeros textos, a decir de los coordinadores, "lo fronterizo como tema de la agenda de análisis de los estudiosos de lo urbano" (p. 9), entendiendo que toda frontera resulta un territorio compartido que involucra "gente, economía y gobiernos en una forma compleja” (p. 84). En el mismo sentido, el autor del cuarto ensayo de esta segunda parte afirma con gran agudeza que las fronteras son un territorio sui generis, cuyo elemento determinante es la binacionalidad (p. 172), fenómeno complementario y abordado por otros autores del libro.

Esta segunda parte comienza con el ensayo de Armando Cantú Alonzo, quien examina el crecimiento económico y social de la frontera noreste México-Estados Unidos, particularmente con el estado de Texas, en el periodo 1848-1940. Mediante un texto de corte histórico, utiliza el enfoque de largo plazo y explica los orígenes sociales de los inmigrantes y el efecto de la ocupación de la población mexicana en la región, refiriéndose incluso a una sociedad y economía binacionales donde los nexos que surgen de esta "relación simbiótica" (p. 84) resultan de gran interés. $\mathrm{Al}$ respecto, destaca que en un primer periodo (1848-1900) los rancheros mexicanos participaron en la etapa clave de la consolidación de Texas para su desarrollo como sociedad agrícola y ganadera. Esto tuvo continuidad en un segundo periodo (1900-1940) donde el idioma, los descendientes, las ligas maritales y de negocios contribuyeron a hispanizar la región.

Si bien el arribo de los ferrocarriles internacionales a Laredo en este segundo periodo facilitó la migración de la población anglo, ${ }^{1}$ incrementando las oportunidades de éstos en las actividades económicas, la inmigración mexicana continuó, debido a diversos acontecimientos cruciales de la historia del país, y garantizó con ello una diversidad ocupacional que fue conformando una creciente clase media.

\footnotetext{
${ }^{1}$ El autor utiliza este término (pp. 77, 79) para referirse a la población de origen estadounidense que se dirigió a la zona fronteriza con el fin de establecer allí su residencia.
} 
Es conveniente comentar que al inicio del relato, el autor advierte que la literatura sobre la frontera de Texas y el noreste mexicano sigue siendo limitada en la actualidad; no obstante, en su desarrollo abunda en referencias con nombres de personajes e historias de caso muy ilustrativas, que hicieron la economía y el desarrollo en general de esta región. Es aquí donde se observa su mayor aporte, aunque cabe comentar que el trabajo habría estado aún más completo con la referencia específica del territorio por medio del uso de herramientas como la cartografía temática.

Por su parte, el ensayo de José Manuel Prieto González reúne una serie de reflexiones en torno al proyecto de construcción del muro fronterizo México-Estados Unidos, y los efectos sociales, políticos, jurídicos y espaciales que ha traído consigo dicha intención política. Comienza con una serie de referencias al significado simbólico de la expresión latina noli me tangere (no me toques, no te acerques a mí), y su traslación a las diferentes actitudes (políticas y civiles) frente a la construcción del muro. De este modo, interpreta al muro fronterizo como materialización de una prohibición, de un sentimiento de desconfianza respecto del otro, el extranjero, el ilegal; observa que no es sino una respuesta a ciertas configuraciones en el imaginario colectivo estadounidense acerca del flujo de migración indocumentada de latinoamericanos (sobre todo mexicanos) en las últimas décadas que, al vincularse con los actos terroristas de 2001 en Nueva York, ha pasado de ser un tema social para convertirse en un asunto de seguridad nacional.

Continuando con una consideración sobre el concepto de mixofobia incorporado por Bauman en los estudios recientes de sociología, como reacción negativa a lo distinto, a la incapacidad de convivir con la variedad de tipos de vida humana que coexisten en las ciudades contemporáneas, Prieto González hace uso de recursos metafóricos y comparaciones entre la construcción del muro, y los requerimientos actuales de grupos de personas que se sienten inseguros en su ciudad. Así explica los efectos espaciales de este temor a lo extraño, por ejemplo, en el uso residencial, donde se ha incrementado la configuración de fraccionamientos cerrados y condominios custodiados que no sólo protegen, sino que además separan a unos residentes de otros.

Por otra parte, es notable la enumeración que realiza el autor sobre la gran cantidad de contradicciones que refleja esta construcción, entre otras: 1) lo "arcaico y primitivo" (p. 108) de la solución, frente a la inversión paralela que se ha comentado se realizaría en alta tecnología (radares terrestres, sensores de movimiento, etc.); 2) el separatismo y xenofobia que supone su construcción frente a los principios de libertad y tolerancia que plantea (o al menos pregona) el sistema democrático estadounidense; 3 ) la uniformidad formal, material y constructiva del muro, en una extensión 
fronteriza de miles de kilómetros; 4) el repudio a un fenómeno que históricamente formó a este territorio, en términos de población: la migración; y 5) una contradicción intrínseca: cuando la civilización ha alcanzado el grado de desarrollo tecnológico más avanzado de la historia, no se plantean soluciones ad hoc con los problemas y desafíos presentes (p. 117).

Con lo anterior y algunos comentarios que recoge de intelectuales, políticos y otros actores importantes, el autor incluso declara que le resulta irrelevante si se construirá o no el muro, toda vez que las intenciones que lo han promovido quedan claras, y reconoce que si bien "la efectividad del instrumento no está plenamente garantizada" (p. 103), éste "ha legitimado una política que criminaliza la inmigración y que, por consiguiente, sólo alcanza a concebir medidas represivas para contenerla" (p. 119).

Los tres textos restantes de esta segunda parte abordan problemáticas socioterritoriales de gran interés: desigualdades socioespaciales (marginación); efectos globales en lo local (fragmentación, dispersión y discontinuidad en el territorio local), y transformaciones de la relación campociudad.

El estudio presentado por J. Milton Aragón Palacios establece una curiosa y hasta novedosa comparación: la diversidad en sistemas ecológicos de dos regiones biogeográficas que convergen en el noreste mexicano, y la heterogeneidad social vinculada al desarrollo de los distintos grupos de población.

El principal argumento del autor es que existe una relación entre los factores ambientales y las localidades con algún grado de marginación, en el sentido de que hay ciertas características fisiográficas que determinan estos grados. Para dar cuenta de ello, el autor recurre metodológicamente a combinar variables ambientales y sociales. Analiza las cubiertas de tipos de suelo y uso de suelo y vegetación modificada de la Comisión Nacional para el Conocimiento y Uso de la Biodiversidad (Conabio), y señala que en el noreste de México existe gran heterogeneidad en términos de ecosistemas terrestres y especies, suelos, climas y elevaciones. En lo referente al aspecto social, utiliza el índice de marginación propuesto por el Consejo Nacional de Población (Conapo) y descubre que al medir este fenómeno en el ámbito de las localidades, se registran grados altos y muy altos en porcentajes considerables (lo que no ocurre si éste se presenta de manera agregada, es decir, en los niveles municipal y estatal).

Para cruzar la información de las variables mencionadas, Aragón se apoya en los sistemas de información geográfica (SIG) y realiza un análisis geográfico y estadístico (prueba de Tukey), que permitió encontrar valores significativos. Precisamente, nota que la mayoría de las localidades que presentan grados altos de marginación se distribuyen en tipos de suelos 
con bajo nivel de productividad o ligados a topografías accidentadas, como las serranías (p. 138).

Con ésta y otras observaciones, el autor se permite cuestionar la validez del índice del Conapo para su aplicación en áreas rurales, dado que los indicadores con los cuales se construye son de corte estructural, relacionados con el acceso a servicios que brinda el Estado cuando, a juicio del autor, el acceso a servicios públicos no es un problema de desarrollo en ellas; así es que en lugares con factores ambientales extremos se demuestra su descontextualización, pues está diseñado para el ámbito urbano. Como conclusión, propone replantear el concepto de marginación, así como su medición para estas zonas.

El trabajo de José Antonio Trujeque Díaz plantea, de manera general, reflexionar acerca de la reacción de las ciudades frente a la globalización, y de manera particular, analizar las relaciones que hay entre los cambios en la economía urbana regional de la frontera noreste de México, con la aparición de periferias populares donde prima una calidad de vida deficiente.

Una preocupación esencial del autor es poder descifrar cómo transforma el capitalismo globalizado a las ciudades, espacios, percepciones y modos de vida; en esta búsqueda, recoge los esfuerzos hechos por la sociología urbana contemporánea (Sassen, Castells, Mollenkopf) para tratar de explicar la segmentación de estructuras socioterritoriales en ciudades y regiones, por medio de las expresiones ciudad dual o ciudad dividida. Para el caso de las ciudades del noreste mexicano, identifica un triple frente de discontinuidades: en la historia urbano-regional, en las políticas de desarrollo para la región noreste y en el proceso de inserción local con la globalización. Estas discontinuidades indican, más que una dualización o una división, una serie de rupturas en el tiempo y en el espacio, por lo que prefiere recurrir al concepto más preciso de ciudades rotas para definir la cuestión en el área de estudio.

Ahora bien, ¿qué relación tiene esto con la formación de las periferias populares en la frontera norte? En este sentido, Trujeque Díaz afirma que el análisis de las relaciones entre globalización y cambios socioterritoriales debe tomar en cuenta a los actores que conducen o resisten los cambios, las políticas públicas que tratan de ajustar las tendencias generales de la economía globalizada de acuerdo con la trayectoria urbano-regional, y los conflictos y alianzas que se forman alrededor de los proyectos de regeneración urbana (pp. 152-153).

A propósito de lo anterior, identifica y caracteriza a los actores y sus dinámicas de migración intraurbana en las ciudades fronterizas (donde la oferta de fuerza de trabajo que la propia economía global ha generado se vuelve un elemento clave), y cómo las mediaciones institucionales 
(intervención de organismos públicos en el reconocimiento de asentamientos irregulares), a través de una matriz de relaciones de propiedad y los circuitos de empobrecimiento en el tiempo, contribuyen a crear periferias populares que aparecen como un "mosaico discontinuo. Islas o fragmentos donde, típicamente, los nuevos habitantes experimentan el descenso en el acceso a servicios urbanos elementales" (p. 176). Con esto, el autor tipifica las relaciones establecidas y observa cómo han afectado la calidad de vida local.

El propósito del ensayo de Alejandro García García es contribuir con reflexiones al urgente reconocimiento sociológico de las transformaciones en la relación campo-ciudad, tratando de vincularlas específicamente con la región noreste de México. En esto, uno de los temas centrales es la cercanía con la frontera de Estados Unidos porque, visto de esta manera, "la relación migratoria campo-ciudad ha pasado de ser regional a nacional e internacional de manera progresiva e intensa" (p. 187).

El discurso se presenta en dos vertientes: de la ciudad al campo y del campo a la ciudad. En el primer caso, el autor reconoce cambios culturales en el campo por la influencia que las comunidades rurales reciben de los medios masivos de comunicación, y los distintos artículos de consumo que los familiares migrantes importan. La población campesina se transforma incluso en un nuevo mercado, donde se advierte el "asedio cultural" (p. 189), no sólo económico.

La segunda mirada permite distinguir cómo el campo se ha ido incorporando a la ciudad a través de actividades como las ferias, el mercado campesino, los rodeos, la música ranchera, expresiones que en un tiempo fueron propias -y prácticamente exclusivas- del ámbito rural.

Como conclusión, el autor señala que esta relación presenta, por una parte, un proceso de hibridación cultural (p. 196) en ambos sentidos, dada la actual red de relaciones económicas y culturales que acercan a lo rural y lo urbano, en un contexto globalizado; por otra, un proceso de creciente dependencia del campo hacia la ciudad, cuando antes se podía hablar de un ámbito territorial autosuficiente.

La tercera parte integra cinco textos, que van de la reflexión teórica al trabajo empírico, y que observan de manera específica diversos procesos en el Área Metropolitana de Monterrey (AMM). Aparecen incluso temas que resultan recurrentes en las metrópolis, pero que siguen trayectorias particulares cuando se aborda un espacio metropolitano determinado: la ocupación de las periferias; el suelo urbano, las dinámicas de uso y el funcionamiento del mercado; los sectores poblacionales desfavorecidos y su atención por medio de programas sociales de combate a la pobreza.

El ensayo de Vladimir Robles es un avance de una investigación teórica (tesis doctoral del autor) cuyo planteamiento central es que el análisis 
urbano requiere incluir ramas de la ciencia relativamente nuevas que buscan explicar lo que significa y cómo se da la transformación urbana. En este sentido, dicha transformación se considera resultado de una confrontación entre los sistemas complejos adaptativos (posible paradigma de investigación, según la revisión de la teoría de sistemas complejos) que explican la evolución urbana y la planificación como instrumento orientador.

Con base en este argumento, el autor se pregunta si el patrón de organización del AMM y su proceso de urbanización se pueden considerar un sistema complejo adaptativo (modelo de Holland), mediante la determinación de los elementos del sistema, y la reflexión teórico-operativa de los mismos en el caso de estudio, Robles confirma esta consideración y propone establecer nuevas líneas de investigación.

El trabajo de Antonio Tamez Tejeda es un estudio de carácter exploratorio sobre la evolución de la morfología urbana de Monterrey desde su génesis a la actualidad, asociándola a la dinámica de los usos de suelo. En esto, el comportamiento de los distintos sectores de población han tenido gran injerencia, orientando e induciendo dicha morfología. Al respecto, el autor hace referencia a las distintas racionalidades que se ponen en juego: la social, la de mercado y la política. Asimismo, recurre a instrumentos gráficos y cartográficos que permiten conocer las preferencias en relación con los polos de atracción actuales en el desplazamiento de los distritos habitacionales y de los corporativos empresariales, lo cual se refleja en los valores inmobiliarios del área metropolitana (p. 236).

De acuerdo con el autor, la evolución de la mancha urbana en Monterrey ha mantenido el patrón de crecimiento habitacional sectorizado (p. 265) de los estratos socioeconómicos, lo cual ha tenido un carácter influyente en la configuración del sistema de centralidades urbanas. Por lo anterior, el rumbo de la morfología urbana del AMM deberá estar basada en el consenso de estas racionalidades, con el objeto de lograr un desarrollo metropolitano equilibrado.

El texto de Roberto García Ortega y Eduardo Souza González versa sobre la caracterización del proceso de metropolización y crecimiento urbano periférico en el AMM a partir de dos ópticas.

1. La primera, fundamentalmente físico espacial, donde identifican la formación del fenómeno de macrocefalia urbana y económica de esta ciudad respecto de la entidad federativa (concentra $85 \%$ de la población de Nuevo León y $87 \%$ del PIB de la entidad); los autores definen cinco etapas-periodos de "maduración" (p. 282) de la metrópoli de 1960 a 2005, desde la expansión hacia los originales municipios periféricos, hoy conurbados, pasando por fenómenos 
urbanos internos, ${ }^{2}$ hasta los procesos de consolidación paulatina y relativa saturación urbana de la metrópoli, donde la mancha urbana sobrepasa en continuidad (y funcionalidad) a los nueve municipios legalmente reconocidos como parte de la misma.

2. La segunda óptica es la demográfica y sugiere que el crecimiento por umbrales o coronas urbanas que conlleva el proceso de metropolización del AMM guarda correlación directa con el grado de envejecimiento de su población y el paulatino desplazamiento de los nuevos núcleos familiares hacia las periferias. En este sentido, los autores reconocen que éste es un proceso que en términos generales se presenta en la evolución de la mayoría de las áreas metropolitanas, denominado ciclo reproductor de la vida de los pobladores de las ciudades (p. 289).

Para concluir, García Ortega y Souza González hacen hincapié en el crecimiento disperso, expansivo, desordenado, sin lógica ni reconocimiento genuino incluso de procesos simultáneos, como la suburbanización y desurbanización, ${ }^{3}$ que caracterizan al AMM; señalan en tal caso que dicho crecimiento, especialmente en las metrópolis de México, debe dejar de ser una especie de botín urbano de unos cuantos y convertirse en un desarrollo urbano y social genuino, autofinanciado y sostenible para la población que las habita (p. 296).

El documento que presenta Jesús Manuel Fitch Osuna forma parte de la investigación doctoral del autor, que analiza las externalidades ambientales en la formación espacial del valor urbano. La delimitación del estudio que se expone corresponde a la zona central del AMM, conocida como anillo intermedio periférico.

El autor comienza el texto con una caracterización y clasificación de las externalidades urbanas; explica que en el caso de los bienes inmuebles, éstas pueden agruparse en tres bloques: externalidades físicas, sociales y desertización urbana, ${ }^{4} \mathrm{y}$ aclara que el efecto de las mismas se basa en el

\footnotetext{
${ }^{2}$ Concentración de funciones comerciales y de servicios en vialidades centrales, consolidación de la conurbación, segregación social, geográfica y económica, especulación urbana en vacíos urbanos intermedios, proliferación de invasión ilegal de predios por colonos pobres, aparición de primeros subcentros comerciales y de servicios en las periferias de altos ingresos, entre otros.

${ }^{3}$ Según los autores, la suburbanización ocurre cuando la periferia alcanza una mayor tasa de crecimiento porcentual anual, respecto de la tasa registrada en la ciudad central. Por su parte, la desurbanización se da cuando la ciudad central observa un despoblamiento relativo o absoluto (p. 290).

${ }^{4}$ Las externalidades físicas se refieren a los valores positivos o negativos que afectan el entorno del inmueble (negativos: contaminación atmosférica y sonora, o bien problemas de tipo sanitario por cercanía a zonas industriales; positivos: proximidad a vías de comunicación, parques o zonas verdes). Las externalidades sociales están representadas por valores positivos o negativos atribuibles a las características demográficas de una zona (clase social y raza, lo que genera segregación residencial de la ciudad). La desertización urbana se explica por el proceso de migración de población de los
} 
bienestar o malestar que no se negocia en el mercado. Otra clasificación refiere a externalidades públicas y privadas, e incluso externalidades bilaterales. ${ }^{5}$

Una vez introducidos estos conceptos, avanza sobre la caracterización del aprovechamiento urbanístico de la zona central del AMM, con el enfoque de las externalidades, y describe los distintos segmentos del mercado inmobiliario, según el tipo de actividad: residencial, comercial, industrial. Aquí hay que destacar la notable utilización de fuentes de información: primero, con informes inmobiliarios de la banca privada, reportes y estudios de mercado comercial, y otros, que en conjunto permiten conformar el panorama de inmuebles, superficies disponibles y localización específica de los mismos en el área de estudio. En segundo lugar, el uso de valores de catastro de Nuevo León, instituciones de valuación estatal y la base de información de la subdirección de Estudios de Posgrado e Investigación de la Facultad de Arquitectura de la UANL, permitieron determinar valores medios a nivel de AGEB, con lo que se obtuvo un mapa del centro metropolitano con valores clasificados por rangos.

De este modo, la comparación de la estructura de dichos valores, y su relación con algunas variables sociales, económicas, de vivienda y territoriales, contribuyen a explicar la formación espacial del valor urbano.

Un comentario que adicionalmente conviene hacer es que no aparecen en este análisis menciones respecto del mercado informal-ilegal de inmuebles, lo cual hace dudar de su existencia en el área de estudio, o pensar si tal vez se trata más bien de un estudio enfocado únicamente al mercado formal-legal de inmuebles.

El último texto pertenece a Socorro Arzaluz Solano, cuyo interés radica en observar la manera en que un programa federal de política social, y su aplicación en el estado de Nuevo León ( en particular en algunas localidades del AMM), beneficia a los sectores más vulnerables de la población. En este caso, la autora refiere los antecedentes del Programa Hábitat y luego indica que, actualmente, parte de la política que se deriva de dicho programa en el estado es la construcción de centros comunitarios; si bien en el país se tiene poco conocimiento de estas experiencias, en esta entidad federativa ha resultado una de las acciones más relevantes y exitosas en materia de política social. De hecho, se ha planteado instalar una red de centros de este tipo, que a la fecha del análisis sumaban veinte.

barrios céntricos de las ciudades - por la obsolescencia funcional de espacio público y privado de estas áreas- hacia otros sitios con mayor calificación urbanística (pp. 303-304).

${ }^{5}$ Las externalidades públicas se dan cuando un bien, o un recurso natural, se usa sin que sea necesario pagar por ello o cuando el consumo que un individuo hace de ese bien o recurso no reduce las posibilidades de su consumo por otros (aire o agua de un acuífero) (pp. 304-305). Cuando una externalidad es bilateral o involucra a pocos individuos, y tanto a los que causan externalidad como aquellos que la sufren son perfectamente identificables, se habla de externalidades privadas. Es el caso tradicional de aquél que quema basura en su jardín molestando con ello al vecino (p. 305). 
Los centros comunitarios (cc) comprenden un espacio físico adecuado, ubicado en zonas de atención (polígonos de pobreza establecidos previamente), que ofrece servicios y apoyo a la población objetivo ( $\mathrm{p}$. 332). En el desarrollo del ensayo se describen cuestiones específicas de funcionamiento, red de actores internos y actividades de uno de ellos, el cc la Alianza, que hasta ahora es el más grande, con más talleres y opciones para la población y mayor población atendida (p. 335).

El objetivo es reflexionar acerca de la influencia de estos centros en la reducción de las situaciones de vulnerabilidad de sus usuarios, toda vez que se analiza un proyecto, a juicio de la autora, difícil de ubicar en las acciones tradicionales de combate a la pobreza, por tratarse de una acción más integral y que observa varias dimensiones del desarrollo humano (p. 342).

A propósito de lo anterior, y dado que el título de este trabajo incluye los temas de pobreza, vulnerabilidad y territorio, hay que advertir cierta omisión respecto de esta última variable y su vínculo con las otras dos, en términos del efecto físico-espacial en el radio de cobertura o influencia del centro, donde los vecinos son los propios usuarios; esto permitiría conocer, por ejemplo, si ha habido mejoras en el parque habitacional, en el espacio público y en las colonias, cuestiones que sin duda se relacionan con el fortalecimiento de las familias y el grupo social en general.

Para concluir esta reseńa, hay que reconocer la complejidad de sujetar a una idea el aporte del libro. Podría decirse precisamente que su mayor contribución es la riqueza y diversidad de enfoques de los textos que lo componen, unos más empíricos y de estudios de caso, otros de corte teórico-reflexivo, pero finalmente todos abordan la región noreste con sus particularidades como preocupación central.

Esto le da especificidad y avanza en el conocimiento de los fenómenos urbano-metropolitanos y su trayectoria en esta zona del país, que se distingue en el contexto mexicano, particularmente en el último siglo, por asuntos políticos y económicos de orden nacional e internacional (TLCAN, flujos migratorios, economía globalizada) que en este territorio han favorecido y desencadenado otros procesos que lo hacen singular.

Recibida: 29 de julio de 2010. Reenviada: 6 de septiembre de 2010. Aceptada: 4 de octubre de 2010.

Carolina Inés Pedrotti

El Colegio Mexiquense, A.C. Correo-e: cpedrotti@cmq.edu.mx 
Carolina Inés Pedrotti. Es maestra en estudios urbanos y regionales por la Universidad Autónoma del Estado de México (UAEM) y arquitecta por la Universidad Nacional de Córdoba (unc, Argentina), con estudios de posgrado en arquitectura, desarrollo y mejoramiento habitacional por la Universidad Mayor de San Simón (umss, Bolivia) y la Universidad de Lund (Suecia). Se desempeña como profesora-investigadora adscrita al Programa Interdisciplinario de Estudios Urbanos y Ambientales (Prourba) de El Colegio Mexiquense, y como profesora de las materias suelo urbano y vivienda en la licenciatura en Administración y Promoción de la Obra Urbana (UAEM). Su línea de investigación es el hábitat urbano (fenómenos socioespaciales vinculados a la vivienda y el suelo urbano destinado al uso habitacional). Entre sus publicaciones destacan: "La seguridad de la tenencia del suelo y la vivienda. Dimensiones del fenómeno desde la perspectiva de la pobreza urbana”, en (varios), Antología del Programa para la Formación de Expertos en Suelo Urbano-Fexsu, 20012006, puec-unam, Lincoln Institute of Land Policy, México, CD-Rom (2006); "Urbanización y política habitacional dominante en el Estado de México... ¿y el derecho a la ciudad?”, en Paula Peyloubet y Laura de Salvo (comps.), Ciencia y tecnología para el hábitat popular. Articulación de politicas intersectoriales: cientifico tecnológicas y de inclusión social, CeveConicet-Nobuko, Buenos Aires, pp. 217-232 (2009); en coautoría, "Las ciudades mexicanas frente a los ODM: la responsabilidad del Estado ante la urbanización descapitalizada y desordenada”, en Carlos Garrocho Rangel (coord.), México y los Objetivos de Desarrollo del Milenio, El Colegio Mexiquense, Zinacantepec, pp. 171-223 (2010). 